\title{
Estimation of the future fracture epidemiology in the patients applying to the emergency department with long short time memory method
}

\author{
$\ddot{O}_{\text {zhan PAZARCI }}{ }^{1}$ (D), Yunis TORUN ${ }^{2}$ (D), Serkan AKKOYUN ${ }^{3, *}$ (iD) \\ ${ }^{1}$ Department of Orthopedics and Traumatology, Sivas Cumhuriyet University, Sivas/Turkey \\ ${ }^{2}$ Department of Electrical and Electronics Eng., Sivas Cumhuriyet University, Sivas/Turkey \\ ${ }^{3}$ Department of Physics, Faculty of Science, Sivas Cumhuriyet University, Sivas/Turkey
}

\begin{abstract}
Operation rooms, human resources and equipment planning are essential for increasing the effectiveness of diagnostic and treatment methods in line with the needs of emergency cases. In this study, 151822 patients admitted to the emergency department (ED) within 3 years were examined in three categories including gender, fracture sites and causes of fracture. However, fracture cases were treated as time series and Long Short Time Memory (LSTM) method was used to estimate the number of future fracture cases. In the learning phase, the number of monthly cases in the next 6 months was estimated using 30-month case numbers. The Root Mean Square Error (RMSE), Mean Absolute Error (MAE) and Mean relative Error (MRE) values of the error rate between the estimated and actual number of cases were given.
\end{abstract}

\section{Article info \\ History:}

Received:01.05.2020

Accepted:12.06.2020

Keywords:

Emergency,

Fractured,

Long Short Time

Memory

\section{Introduction}

With the changing living conditions, trauma and fracture patterns also change [1]. Epidemiological studies are used to identify them and to plan health services. The ability to act based on knowledge is important in today's world. In the field of health, patient information creates an intense accumulation of data. There are several methods for proper evaluation and analysis of this data. In recent years, machine learning was introduced in the medical applications and showed its remarkable efficiency in clinical diagnosis and decision support [2]. Presently, the recurrent neural network (RNN) was well employed in solving time series prediction problems and achieved prominent results in many fields. Several variants of RNN have been developed, and among them, long short-term memory (LSTM) network is one of the most popular variants. LSTM learns longterm dependencies by incorporating a memory cell that can preserve state over time [3].

The answer to the question "What will happen in the future?" is important in providing health services. The artificial neural network method is also emphasized in the literature in predicting future patients [4]. Artificial neural networks have also started to be used in the field of orthopedics [5]. Our study stands out with the use of this method to predict future fracture patients. Current orthopedic literature in this area is generally in the direction of fracture diagnosis from patient images [6]. There are also studies suggesting the diagnosis of a fracture in automated systems with deep learning methods [7]. The general challenge in healthcare today is that physicians have access to a large amount of data about patients, but they have little time and tools to analyze them. The intelligent clinical decision supports in this direction are important for health service delivery planning, cost calculation and personnel distribution planning. These methods are used in future patient estimates [8]. There are few studies using the LSTM (Long Short Time Memory) model to predict patients who have had a heart attack $[9,10]$. LSTM has been using for a long time in solving forecasting problems and analyzing big data [11]. However, no studies on the future prediction of orthopedic cases admitted to the emergency departments have been found in the recent literature.

With the presented study, using the LSTM method, 
complex and heterogenic patient information and future patient prediction were made. According to this study, patients with limb fractures were examined epidemiologically from 151.822 patients who have applied to the emergency department in the last 3 years. In the study, it was aimed to present the fractures epidemiologically according to age, gender groups, distribution throughout the year, fracture region - shape and causes of the accident. Besides, it was tried to predict future case projections using LSTM method. The availability of LSTM in this area has been studied.

\section{Materials and Methods}

\subsection{Data collecting}

Among 151.822 patients who applied to the emergency department of the Sivas Cumhuriyet University Medical Faculty in 2011-2013, 2.105 patients who applied for the extremity fracture were included in the study. The data were obtained by scanning backward from the registry. Age, gender, diagnosis and causes of accidents were noted. The patients were divided into four groups according to their ages as $18-40,40-65,65-80$ and greater than 80 old. The distribution of fractures according to gender was examined. Open and closed fracture rates were noted. The broken regions are listed as shown in Table 1. The data analysis was done in SPSS (Ver.23) program. Data were examined by considering percentage distribution, frequency and arithmetic means.

\subsection{Long short time memory (LSTM)}

Although Neural Network (NN) has achieved success in many applications, it does not achieve the desired accuracy in time series prediction. Recurrent Neural network (RNN) architecture, which enables the addition of the effect of past data to the next output in the time series prediction, reaches higher accuracy than $\mathrm{NN}$ in this series. However, if past inputs are connected to a longer sequence, performance rates decrease due to the short memory of RNNs. Long Short-Term Memory, LSTM architecture, which has been developed as a different version of RNN, allows the creation of longer past input-output relations [12]. In this study, Vanilla LSTM architecture by Gers and Schidhuber was used in LTSM method that has many versions [13]. The LTSM block consists of three gates named input gate, output gate, gate candidate and forget gate and a sub-block. The main idea of LSTM is that the current input of each block, $x_{t}$ and $h_{t-1}$, which represents the short-term output of the previous block and the long-term output from the previous blocks, takes $c_{t-1}$ into account for obtaining the block output. An LTSM block generates the short term $h_{t}$ and long term $c_{t}$ belonging to that block.

The output of the forget gate is a nonlinear function of the current input with the state from the previous block, expressed by the sigmoid activation function;

$f_{t}=\sigma_{g}\left(W_{f} x_{t}+U_{f} h_{t-1}+b_{f}\right)$

where $f_{t}$ is the output of forget gate, $b_{f}$ is the bias for forget gate, $\sigma_{g}$ denotes gate sigmoid activation function. $W_{f}$ and $U_{f}$ corresponds forget gate coefficients. This output is between 0 and 1 , and generates information on whether to be processed in the previous state in the current block or not. The input gate generates an output to calculate $c_{t}$ with a sigmoid activation function of $x_{t}$ and $h_{t-1}$ inputs.

$i_{t}=\sigma_{g}\left(W_{i} x_{t}+U_{i} h_{t-1}+b_{i}\right)$

where $i_{t}$ is the output of the input gate, $b_{i}$ is the bias for forget gate, $\sigma_{g}$ denotes gate sigmoid activation function. $W_{i}$ and $U_{i}$ corresponds to input gate coefficients. The output gate generates information about whether the information in the previous door will be moved to the next block or not.

$$
o_{t}=\sigma_{g}\left(W_{o} x_{t}+U_{o} h_{t-1}+b_{o}\right)
$$

where $o_{t}$ is the output of the input gate, $o_{i}$ is the bias for forget gate, $\sigma_{g}$ denotes gate sigmoid activation function. $W_{0}$ and $U_{0}$ correspond to input gate coefficients. The output gate generates information about whether the information in the previous door will be moved to the next block or not. If the long term memory information of the current block is $c_{t}$ the cell candidate block output is generated with $z_{t}$.

$$
\begin{aligned}
& z_{t}=\tanh \left(W_{z} x_{t}+U_{z} h_{t-1}+b_{z}\right) \\
& c_{t}=f_{t} \times c_{t-1}+i_{t} \times z_{t} \\
& h_{t}=o_{t} \times \tanh \left(c_{t}\right)
\end{aligned}
$$

\section{Results and Discussion}

The recent Covid-19 epidemic also showed us that the estimation of the volume of patients is very important for planning for health service. Although 
some operations could be postponed to reduce the effect of the outbreak by reducing the hospital burden with lower patient acceptance, fractured patients treatment could not be postponed because of the urgency of treatment. Our study shows that the method we use can be used in other diagnostic fields as well.

The extraction of future projections of the cases with LSTM was carried out by coding deep learning codes in the Matlab programming language. The total of 36month case data is divided into two parts: 30 months for the training and last 6 months cases for the estimation. The error between the LTSM estimation and the number of cases for each month in 6 months and the actual number of cases was used to measure the performance of the future estimator presented.

\subsection{Performance indexes}

To asses prediction performance of LSTM based case prediction, three performance indexes were used. Mean absolute error (MAE) was calculated as

$$
M A E=\frac{1}{n} \sum_{i=1}^{n}\left|f_{i}-\widetilde{f}_{i}\right|
$$

Mean relative error (MRE) is calculated as

$$
M R E=\frac{1}{n} \sum_{i=1}^{n} \frac{\left|f_{\mathrm{i}}-\hat{f}_{\mathrm{l}}\right|}{f_{\mathrm{i}}}
$$

Root mean square error (RMSE) is calculated as

$$
R M S E=\left[\frac{1}{n} \sum_{i=1}^{n}\left|f_{i}-\widetilde{f_{i}}\right|^{2}\right]^{\frac{1}{2}}
$$

where $f_{i}$ is the actual value, $\hat{f}_{i}$ is the predicted value for $n$ instants.

\subsection{Simulation environment and result}

The prediction model was set up with the Matlab Neural Network Toolbox Results (Matlab R2018a). More than one scenario has been established for the estimation of the cases. These scenarios are as follows.

Scenario 1: Estimation of the number of cases by gender for the next 6 months.

Scenario 2: Estimation of the number of cases for the next 6 months according to the occurrence of the case.

Scenario 3: Estimation of cases by fracture site classes for the next 6 months according to the fracture sites occurring in cases.

Open fractures constituted $8.8 \%$ (186 of all cases) of all fractures that applied to the emergency department. These fractures are; Fractures were $46.2 \%$, tibia shaft $11.8 \%$, ankle $7.5 \%$, and tibia distal $6.5 \%$ fractures. The leading cause of open fractures was a direct impact to bone $73.7 \%$. In our study, the spine, pelvis, talus, calcaneus and femur shaft open fractures were not observed.

According to the first scenario, 6-month estimation of the number of cases of men and women was made with LSTM, and their performance is shown in figure 1 and 2, respectively. In the estimation made for women, it is seen that there are approximately 20 cases between the number of cases realized in the first two months and the number of cases estimated, but the difference between the number of cases estimated for each month and the number of cases realized is less than 10. In the prediction of cases with male gender, a better estimation performance was obtained compared to women. Within the six-month forecast period, each case number error was estimated with \pm 10 errors, and the RMSE value was found to be 9.8.
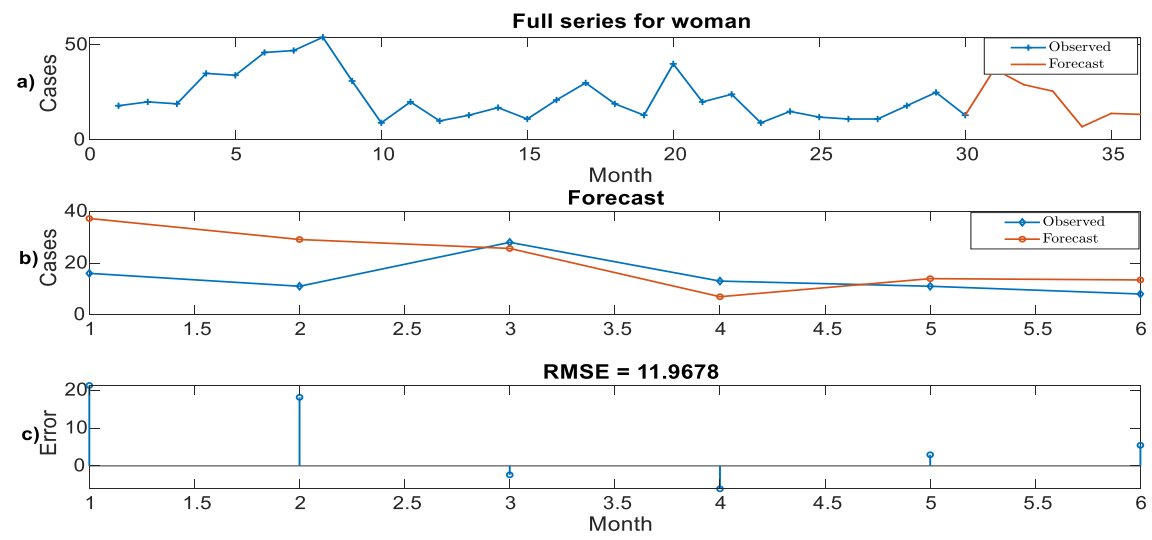
Figure 1. a) Full series of the case for woman (a), Predicted and actual case numbers (b), Prediction error for each month (c)
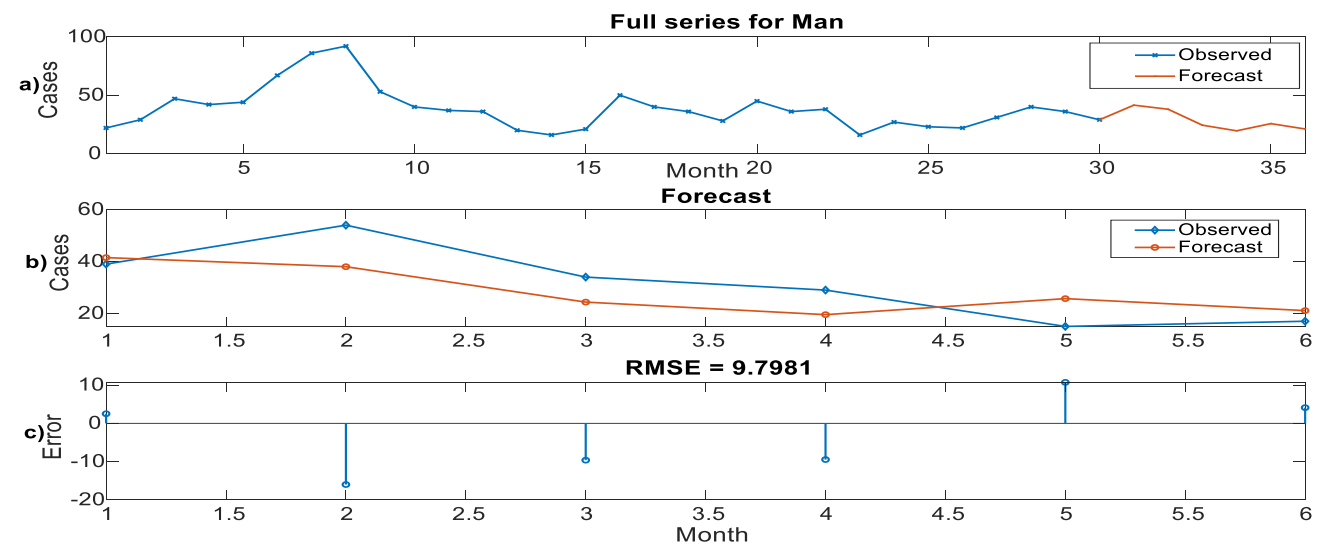

Figure 2. Full series of the case for man (a), Predicted and actual case numbers (b), Prediction error for each month (c)

In scenario 2, a classification was made according to the reason for the occurrence of the cases and an attempt was made to estimate the number of 6-month cases according to these classes. According to the reason of the broken cases, the cases were defined in eight classes which are a) normal falling, b) falling from the ladder, c) falling from a high place, d) the impact took as a result of the fight, e) sports injury, f) traffic accident, g) stress and h) others not in another class. The number of cases for 30 months and the number of estimates and actual cases for the next 6 months are shown in Figure 3. The graph with the blue line shows the number of cases that occurred, while the red line shows the number of cases that were estimated.

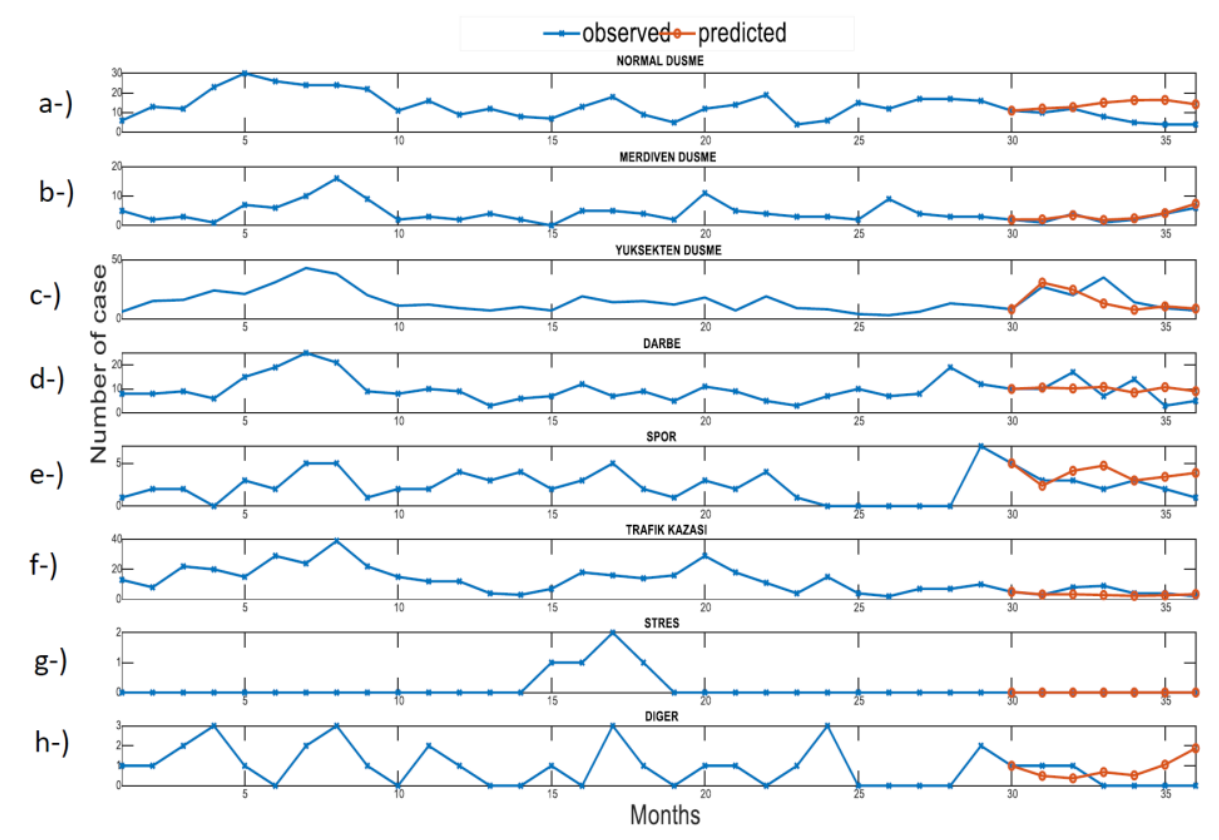

Figure 3. Observed and predicted case according to the cause formation

In scenario 3, cases are divided into two main classes as Upper limp and Lower limp according to fracture areas. Upper limb identifies cases to separate fracture sites and these fractures are Clavicula, Scapula, Humerus, Forearm, Handphalanx, Scaphoid, Spine, and Pelvis fractures.
Lower Limb fractures are classified according to 9 lower fracture regions and they are Femur, Patella, Tibia, Tarsal bones, Talus, Calcaneus, Foot phalanx, Ankle, Metatars fractures. In Table1, estimation performances of the number of cases according to the 6-month fracture region 
are given. RMSE, MAE and MREs of the prediction errors of each major fracture region and the specific region where the fracture occurs are indicated in the table. Average RMSE, MAE and MRE values of each class were calculated as $1.053,0.839$ and 0.358 , respectively.

Table 1. Performance indices of 6-month forecasts by broken region

\begin{tabular}{|c|c|c|c|c|}
\hline Broken region & & RMSE & MAE & MRE \\
\hline \multicolumn{5}{|l|}{ UPPER LIMB } \\
\hline Clavicula & & 5,441 & 4,576 & 1,052 \\
\hline Scapula & & 1,463 & 1,266 & 0,476 \\
\hline \multirow[t]{3}{*}{ Humerus } & Proximal & 1,290 & 1,045 & 0,135 \\
\hline & Shaft & 1,460 & 1,147 & 0,806 \\
\hline & Distal & 3,869 & 2,719 & 0,572 \\
\hline \multirow[t]{3}{*}{ Forearm } & Proximal & 1,311 & 1,085 & 0,274 \\
\hline & Shaft & 1,742 & 1,090 & 0,402 \\
\hline & Distal & 2,119 & 1,841 & 0,137 \\
\hline Hand-phalanks & & 7,700 & 7,031 & 1,231 \\
\hline Schaphoid & & 1,856 & 1,538 & 0,000 \\
\hline Spine & & 0,545 & 0,351 & 0,934 \\
\hline Pelvis & & 3,118 & 2,738 & 0,188 \\
\hline \multicolumn{5}{|l|}{ LOWER LIMB } \\
\hline \multirow[t]{3}{*}{ Femur } & Proximal & 4,143 & 3,298 & 0,420 \\
\hline & Shaft & 2,403 & 1,746 & 0,836 \\
\hline & Distal & 1,041 & 0,810 & 0,963 \\
\hline Patella & & 0,806 & 0,702 & 0,361 \\
\hline \multirow[t]{3}{*}{ Tibia } & Proximal & 1,489 & 1,178 & 0,933 \\
\hline & Shaft & 1,221 & 1,176 & 0,028 \\
\hline & Distal & 1,617 & 1,497 & 0,555 \\
\hline Tarsal bones & & 0,582 & 0,454 & 0,996 \\
\hline Talus & & 0,451 & 0,304 & 0,415 \\
\hline Calcaneus & & 0,730 & 0,373 & 0,000 \\
\hline Foot phalanks & & 0,378 & 0,285 & 0,083 \\
\hline Ankle & & 1,118 & 0,935 & 0,041 \\
\hline Metatars & & 1,695 & 1,322 & 0,769 \\
\hline Mean & & 1,053 & 0,839 & 0,358 \\
\hline
\end{tabular}

\section{Conclusions}

Knowing the epidemiological distribution and rates of fractures is important for preventive medicine as well as health service delivery and planning. According to our study, fall-related fractures in childhood are more common, while osteoporotic fractures caused by minor trauma are observed in the foreground in elderly patients. Hand fractures that occur as a result of the direct impact on bone are more common in adults who form an active workforce. In long bone open fractures, which are difficult and costly to treat, tibial fractures are in the first place. Despite all preventive activities, motor vehicle accidents are the leading cause of fractures. Epidemiological studies should be carried out at certain intervals according to changing living conditions and human activities. Removing future projections of fracture cases is very important for the programming of emergency and orthopedic clinical human resources, medical equipment and patient services. In this study, for the first time, a 6-month case number estimation from 30month case numbers was made by using LSTM, which is one of the deep learning methods. Since the shape of the fracture affects the planning of the health service provided, the causes of the fracture again, satisfactory results were obtained in 3 different scenarios, according to the gender, the occurrence of the case and the fracture regions formed in the cases, 
according to the fracture class classes for the next 6 months.

\section{Conflicts of interest}

The outhors state that did not have conflict of interests

\section{References}

[1] Kosuge D, Barry M. Changing trends in the management of; children's fractures. Bone and Joint Journal, 97(4) (2015) 442-448.

[2] Court-Brown CM, Caesar B. Epidemiology of adult fractures: A review. Injury, 37(8) (2006) 691-697.

[3] Xia J, Pan S, Zhu M, Cai G, Yan M, Su Q, et al. A Long Short-Term Memory Ensemble Approach for Improving the Outcome Prediction in Intensive Care Unit. Computational and Mathematical Methods in Medicine, 2019 (2019) 1-10.

[4] Pham T, Tran T, Phung D, Venkatesh S. Predicting healthcare trajectories from medical records: A deep learning approach. Journal of Biomedical Informatics, 69(2) (2017) 18-29.

[5] Olczak J, Fahlberg N, Maki A, Razavian AS, Jilert A, Stark A, et al. Artificial intelligence for analyzing orthopedic trauma radiographs: Deep learning algorithms - are they on par with humans for diagnosing fractures? Acta Orthopaedica, 88 (2017) 581-586.

[6] Lindsey R, Daluiski A, Chopra S, Lachapelle A, Mozer M, Sicular S, et al. Deep neural network improves fracture detection by clinicians. Proceedings of the National
Academy of Sciences of the United States of America, 115(45) (2018) 11591-11596.

[7] Cheng CT, Ho TY, Lee TY, Chang CC, Chou $\mathrm{CC}$, Chen $\mathrm{CC}$, et al. Application of a deep learning algorithm for detection and visualization of hip fractures on plain pelvic radiographs. European Radiology, 29 (2019) 5469-5477.

[8] Choi E, Bahadori MT, Schuetz A, Stewart WF, Sun J. Doctor AI: Predicting Clinical Events via Recurrent Neural Networks. JMLR Workshop and Conference Proceedings 2015.

[9] Maragatham G, Devi S. LSTM Model for Prediction of Heart Failure in Big Data. Journal of Medical Systems, 43 (2019) 111.

[10] Golas SB, Shibahara T, Agboola S, Otaki H, Sato J, Nakae T, et al. A machine learning model to predict the risk of 30-day readmissions in patients with heart failure: a retrospective analysis of electronic medical records data. BMC Medical Informatics and Decision Making, 18 (2018) 44.

[11] James AP. Deep Learning Classifiers with Memristive Networks. vol. 14. Cham: Springer International Publishing; 2020.

[12] Hochreiter S, Schmidhuber J. Long ShortTerm Memory. Neural Computation 1997.

[13] Gers FA, Schmidhuber J. Recurrent nets that time and count. Proceedings of the IEEEINNS-ENNS International Joint Conference on Neural Networks. IJCNN 2000. Neural Computing: New Challenges and Perspectives for the New Millennium, IEEE, 3 (2000) 18994. 